\title{
Resveratrol-4-O-D-(2'-galloyl)-glucopyranoside exerts an anticancer effect on leukemia cells via inducing apoptosis
}

\author{
PU CHEN, BEILI WANG, BAISHEN PAN and WEI GUO
}

\begin{abstract}
Department of Laboratory Medicine, Zhongshan Hospital, Fudan University, Shanghai 200032, P.R. China
\end{abstract}
Received March 4, 2015; Accepted December 15, 2015

DOI: $10.3892 / \mathrm{mmr} .2016 .4777$

\begin{abstract}
The aim of the present study was to investigate the anticancer effects of resveratrol-4-O-D-(2'-galloyl)-glucopyranoside (REG) on leukemia and the mechanism underlying its effects. Three leukemia cell lines (HL-60, Jurkat and U937) were used in this study. A Cell Counting kit- 8 assay was performed to evaluate the anti-proliferative activity of REG on leukemia cell lines, and flow cytometric analysis was used to detect REG-induced apoptosis. In addition, western blot analysis was conducted to detect the levels of apoptosis-related proteins including, cytochrome $c$, cleaved (c)-caspases-3 and -9, B-cell lymphoma 2 (Bcl-2) and Bcl-2-associated protein $\mathrm{x}$ (Bax). Finally, a HL-60 cell xenograft model in nude mice was used to evaluate the antitumor effect of REG on leukemia in vivo. The present results indicated that REG can significantly inhibit the proliferation of HL-60, Jurkat and U937 cell lines in a concentration- and time-dependent manner. The half maximal inhibitory concentration values were 38.4, 49.1 and $48.2 \mu \mathrm{g} / \mathrm{ml}$ for HL-60, Jurkat and U937 cells, respectively. Furthermore, flow cytometric analysis demonstrated that REG can induce the apoptosis of HL-60 cells, as well as increase the levels of cytochrome $c$, c-caspases-3 and -9 , and Bax, as well as downregulate the expression of $\mathrm{Bcl}-2$. In vivo, $\mathrm{REG}$ was found to possess a marked anticancer effect on leukemia. In combination, the present results indicated that REG exerts significant anticancer effects on leukemia in vivo and in vitro through the induction of apoptosis.
\end{abstract}

\section{Introduction}

Leukemia is one of the leading causes of malignant cancer-related mortality in males and females, and despite the efforts made to improve its treatment and diagnosis, the mortality rates remain high to date (1). Currently, the

Correspondence to: Dr Wei Guo, Department of Laboratory Medicine, Zhongshan Hospital, Fudan University, 111 Yixueyuan Road, Shanghai 200032, P.R. China

E-mail: weiguofd2010@126.com

Keywords: anticancer, leukemia, apoptosis, Polygonum cuspidatum, resveratrol-4-O-D-(2'-galloyl)-glucopyranoside, HL-60 predominant therapeutic regimens for the treatment of leukemia are chemotherapy-based, due to the fact that the majority of the adult patients with leukemia are incompatible for bone marrow transplantation. Chemotherapy-based treatments, however, are associated with severe side effects $(2,3)$, and therefore, finding novel, safe and effective therapeutic regimens for leukemia is imperative. Traditional Chinese medicines are used for the treatment of various diseases, and a number of these agents have been considered as potential agents for cancer treatment or prevention (4-7).

Resveratrol-4-O-D-(2'-galloyl)-glucopyranoside (REG) is one of the predominant stilbene glycosides isolated from Polygonum cuspidatum (8). No pharmacological activity of REG has been reported so far, however, preliminary experiments demonstrated potential anticancer effects in vivo. The present study investigated the effect of REG on leukemia cells, and the findings demonstrated that it exerts marked anticancer effects on leukemia through the induction of apoptosis.

\section{Materials and methods}

Chemicals and reagents. Resveratrol-4-O-D-(2'-galloyl) -glucopyranoside (Fig. 1) was purchased from Dingrui Chemical Ltd. (Shanghai, China). Cell Counting kit-8 (CCK-8) was obtained from Dojindo Biochem (Shanghai, China), dimethyl sulfoxide (DMSO) from Sigma-Aldrich (St. Louis, MO, USA), and RPMI 1640 media and fetal bovine serum (FBS) from Invitrogen, Thermo Fisher Scientific, Inc., (Waltham, MA, USA). Monoclonal rabbit antibodies against $\beta$-actin (cat. no. 49701), cytochrome $c$ (cat. no. 4280s), cleaved (c)-caspases-3 (cat. no. 9664s) and -9 (cat. no. 9509s), B-cell lymphoma 2 (Bcl-2; cat. no. 2870s) and Bcl-2-associated protein x (Bax; cat. no. 5023s) were purchased from Cell Signaling Technology Inc. (Denver, MA, USA). An Annexin V-fluorescein isothiocyanate (FITC) propidium iodide (PI) kit was purchased from Beyotime Institute of Biotechnology (Haimen, China). All other chemicals used in the present study were of analytical grade.

Animals. Nude mice ( $\mathrm{n}=20$; age, 5-6 weeks) were obtained from the Shanghai SLRC Laboratory Animal Company (Shanghai, China). The mice were maintained at $21 \pm 1^{\circ} \mathrm{C}$ under a 12-h light/dark cycle with access to standard food pellets and water ad libitum. All animal treatments were conducted in strict accordance with the international ethical guidelines 
<smiles>O=C(OCC1OC2OC(O)C(O)C(O)C(O)C1O2)c1cc(O)cc(/C=C/c2ccc(O)cc2)c1</smiles>

Figure 1. Chemical structure of resveratrol-4-O-D-(2'-galloyl)-glucopyranoside.

and the Guide for the Care and Use of Laboratory Animals by the National Institutes of Health (9). The experiments were approved by the Animal Experimentation Ethics Committee of Fudan University (Shanghai, China).

Cell culture and CCK-8 assay. HL-60, Jurkat and U937 human leukemia cell lines were purchased from the American Type Culture Collection (Manassas, VA, USA). The cells were cultured in RPMI-1640 medium supplemented with $10 \% \mathrm{FBS}, 100 \mathrm{U} / \mathrm{ml}$ penicillin and $100 \mu \mathrm{g} / \mathrm{ml}$ streptomycin (Sigma-Aldrich). Cells were sub-cultured until they reached the logarithmic growth phase at $7^{\circ} \mathrm{C}$ in $5 \% \mathrm{CO}_{2} / 95 \%$ air. Then, the CCK assay was performed to evaluate the anti-proliferative activity of REG on leukemia cell lines as previously described (10).

Flow cytometry for the detection of apoptosis. HL-60 cells were harvested, washed with phosphate-buffered saline and stained using a commercial Annexin V-FITC/PI kit according to the manufacturer's protocol. Cell apoptosis was detected by flow cytometric analysis on a FACSCalibur ${ }^{\mathrm{TM}}$ flow cytometer (BD Biosciences, Franklin Lakes, NJ, USA). The percentage of cells undergoing early apoptosis was indicated by Annexin V-positivity and PI-negativity, and that of cells undergoing late apoptosis was indicated by Annexin V-positivity and PI-positivity.

Western blot analysis. Following total protein extraction from cells using mammalian protein extraction reagents (Beyotime Institute of Biotechnology), the protein concentration was determined using the Enhanced Bicinchoninic acid Protein Assay Reagent (Beyotime Institute of Biotechnology). Subsequently, $40 \mu \mathrm{g}$ protein samples were separated by $12 \%$ sodium dodecyl sulfate polyacrylamide gel electrophoresis (Beyotime Institute of Biotechnology), and transferred onto polyvinylidene difluoride membrane (EMD Millipore, Billerica, MA, USA). The membranes were blocked with $5 \%$ fat-free dry milk in $1 \mathrm{X}$ Tris-buffered saline containing $0.05 \%$ Tween 20 for $2 \mathrm{~h}$, and incubated with monoclonal primary antibodies (diluted $1: 1,000$ ) at $4^{\circ} \mathrm{C}$ for $10 \mathrm{~h}$. The membrane was washed with TBST buffer [Sangon Biotech Co., Ltd., Shanghai, China; $20 \mathrm{M}$ Tris- $\mathrm{HCl}$ (pH 7.4), $150 \mathrm{M} \mathrm{NaCl}$ and $0.1 \%$ Tween 20 and protein bands were incubated with goat anti-rabbit horseradish peroxidase-conjugated monoclonal antibody (diluted, 1:2,000; cat. no. A0208; Beyotime Institute of Biotechnology) for $2 \mathrm{~h}$ at room temperature, and subsequently detected by chemiluminescence (Beyotime Institute of Biotechnology) using a ChemiDoc ${ }^{\mathrm{TM}}$ XRS imaging system
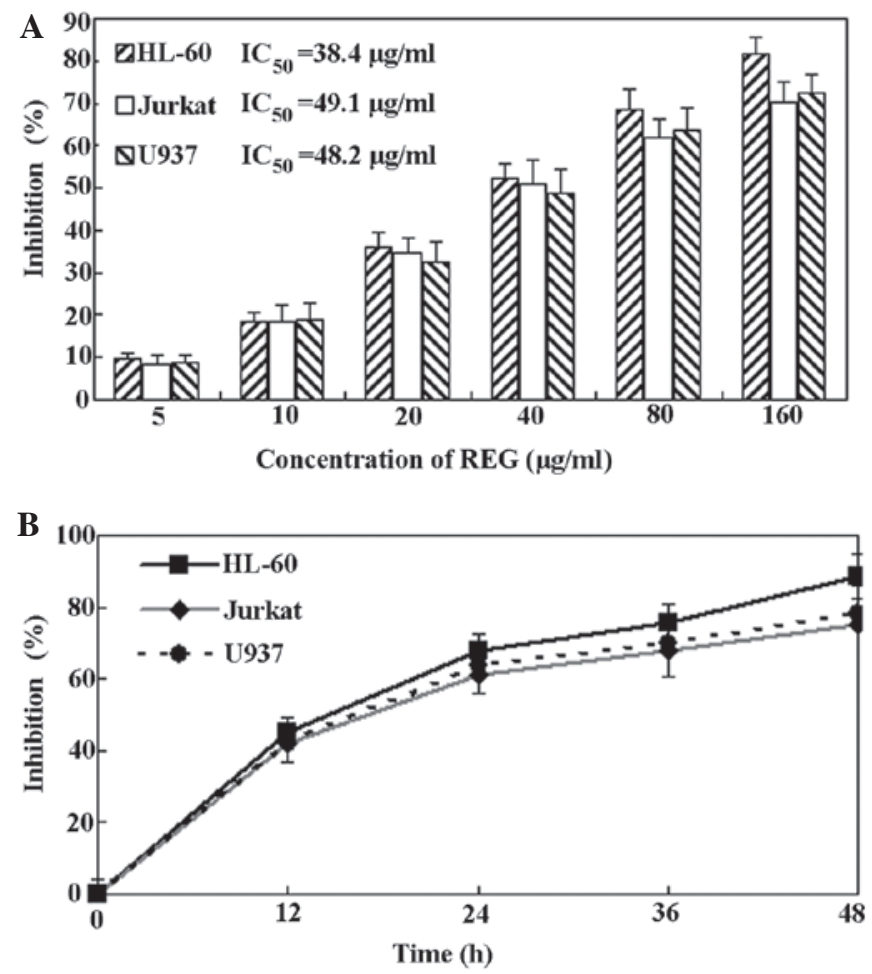

Figure 2. Anti-proliferative activity of REG on leukemia cells (A) Concentration-dependent effect of REG on leukemia cell proliferation. Cell lines were treated with low-to-high concentrations of REG $(5,10,20$, 40,80 and $160 \mu \mathrm{g} / \mathrm{ml}$ ) for $24 \mathrm{~h}$, and then a CCK-8 assay was performed and the values calculated. (B) Time-dependent effect of REG on leukemia cells Cell lines were treated with REG $(80 \mu \mathrm{g} / \mathrm{ml})$ for 12,24 and $48 \mathrm{~h}$, and then a CCK-8 assay was performed. Data are presented as the mean \pm standard deviation (n=4). REG, resveratrol-4-O-D-(2'-galloyl)-glucopyranoside; CCK-8, cell counting kit-8; $\mathrm{IC}_{50}$, half maximal inhibitory concentration values.

(Bio-Rad Laboratories, Inc., Hercules, CA, USA). To measure protein loading, antibodies directed against $\beta$-actin were used.

Xenograft assay in vivo. The mice were divided into the REG treatment and control groups ( $\mathrm{n}=8 / \mathrm{group})$. All mice received a subcutaneous injection of HL-60 cells $\left(2 \times 10^{6} /\right.$ mouse) in the right flank. When the tumors grew to $\sim 2-3 \mathrm{~mm}$ in diameter, the REG treatment group received an intraperitoneal injection of REG $(40 \mathrm{mg} / \mathrm{kg} / \mathrm{day})$ and the control group received an equal volume of solvent control (0.5\% DMSO). The mice from the two groups remained under observation for 15 days, and the tumor sizes were measured every 5 days. The tumor diameters were determined using a Vernier caliper (Bangsheng Equipment, Shanghai, China), and the tumor 

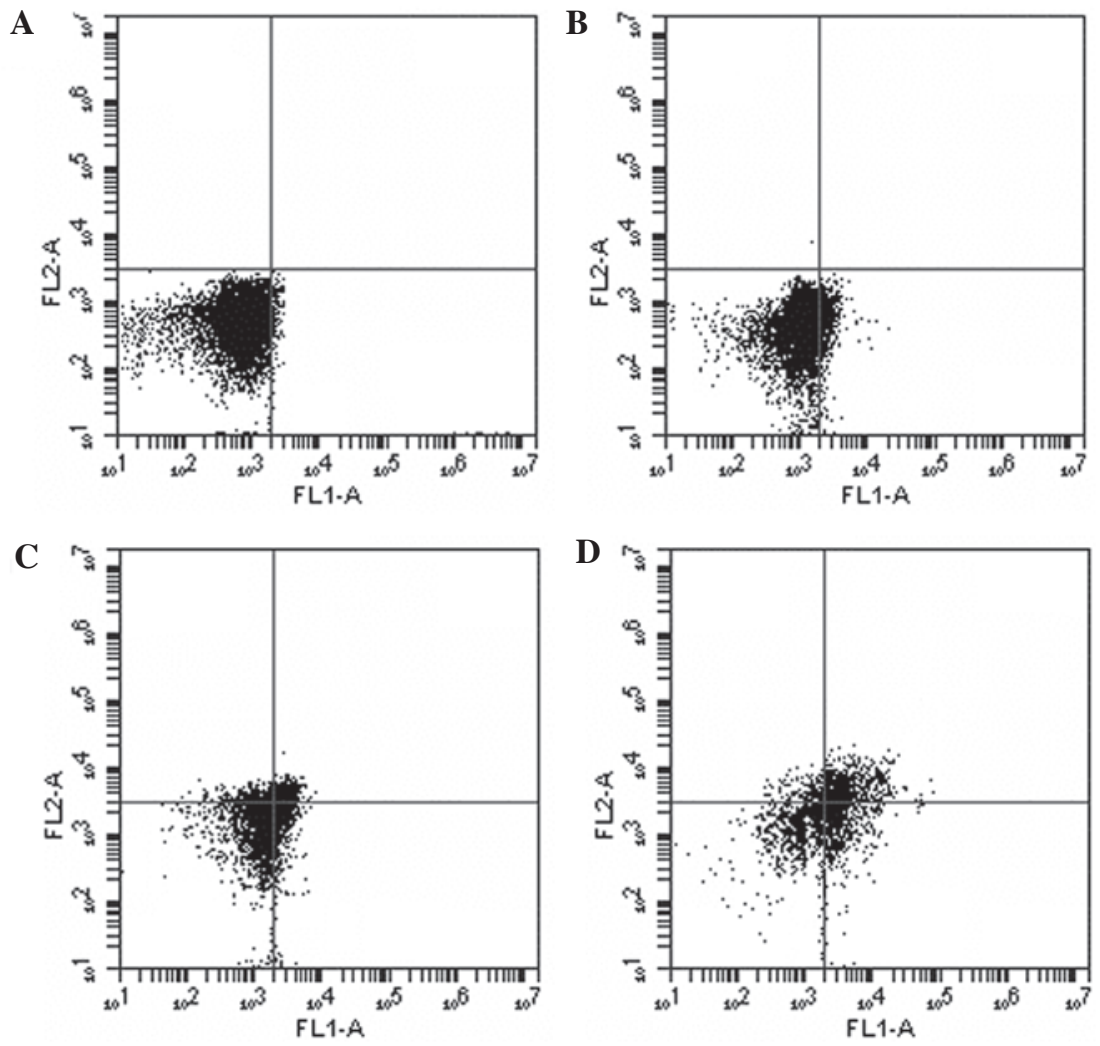

Figure 3. Flow cytometric analysis for the detection of apoptosis of HL-60 cells following exposure to REG. Cell lines were (A) left untreated or treated with (B) 20 , (C) 40 or (D) $80 \mu \mathrm{g} / \mathrm{ml} \mathrm{REG}$ for $24 \mathrm{~h}$, and then flow cytometry was performed to determine apoptosis using Annexin-V/propidium iodide double-staining. REG, resveratrol-4-O-D-(2'-galloyl)-glucopyranoside.


Figure 4. Effect of REG on the protein expression of cytochrome $c$ and c-caspases-3 and -9 in HL-60 cells. HL-60 cells were exposed to REG (20, 40 and $80 \mu \mathrm{g} / \mathrm{ml}$ ) for $24 \mathrm{~h}$, and then the protein levels were measured using western blotting. The data are presented as the mean \pm standard deviation $(\mathrm{n}=4)$. ${ }^{*} \mathrm{P}<0.05$ and ${ }^{* * *} \mathrm{P}<0.01$ vs. control. REG, resveratrol-4-O-D-(2'-galloyl)-glucopyranoside; c-caspase, cleaved-caspase.

volumes were then calculated according to the formula (11): Volume $=\left(\right.$ width $^{2} \mathrm{x}$ length $) / 2$. All the animals were sacrificed by anesthesia with $50 \mathrm{mg} / \mathrm{kg}$ pentobarbital sodium
(Sigma-Aldrich) immediately after 15 days of observation, and the tumors tissues were collected and homogenized for western blot analysis. 

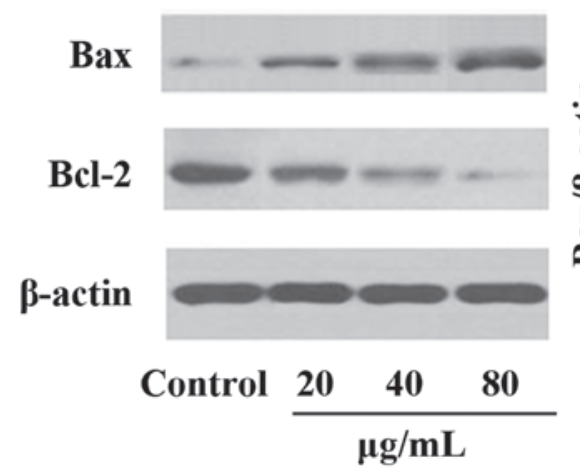

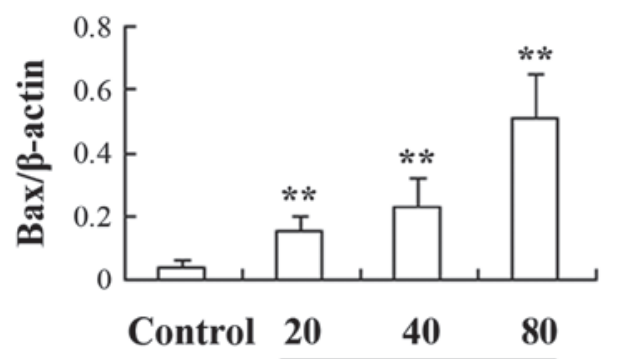

$\mu \mathrm{g} / \mathrm{mL}$

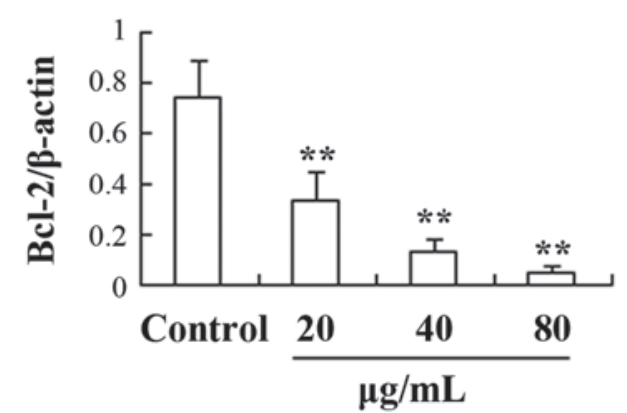

Figure 5. Effect of REG on the protein expression of Bcl-2 and Bax in HL-60 cells. HL-60 cells were exposed to REG (20, 40 and $80 \mu \mathrm{g} / \mathrm{ml})$ for $24 \mathrm{~h}$, and then the protein levels were measured using western blotting. The data are presented as the mean \pm standard deviation $(\mathrm{n}=4)$. ${ }^{*} \mathrm{P}<0.05$ and ${ }^{* *} \mathrm{P}<0.01 \mathrm{vs}$. control. $\mathrm{REG}$, resveratrol-4-O-D-(2'-galloyl)-glucopyranoside; Bcl-2, B-cell lymphoma 2; Bax, Bcl-2-associated protein x.

Statistical analysis. The significant differences between the groups were determined using Student's t-test and analyzed by SPSS software for Windows (version 18.0; SPSS, Inc., Chicago, IL, USA). The results are presented as the mean \pm standard deviation. $\mathrm{P}<0.05$ was considered to indicate a statistically significant difference.

\section{Results}

REG exhibits an anti-proliferative effect on leukemia cell lines. HL-60, Jurkat and U937 leukemia cell lines were treated with REG at concentrations of 5-160 $\mu \mathrm{g} / \mathrm{ml}$, and the cytotoxic activity of REG was evaluated using a CCK-8 assay. The results indicated that REG exerted a marked anti-proliferative effect on the three leukemia cell lines in a concentration and time-dependent manner (Fig. 2). The half maximal inhibitory concentration $\left(\mathrm{IC}_{50}\right)$ values of REG for HL-60, Jurkat and U937 ell lines were 38.4, 49.1 and $48.2 \mu \mathrm{g} / \mathrm{ml}$, respectively. These results indicated that REG can significantly inhibit proliferation in leukemia cells in a concentration- and time-dependent manner. In addition, the HL-60 cell line was selected for the rest of the experiments as REG exhibited the most marked anti-proliferative effect on this cell line.

Flow cytometric analysis results. In order to confirm whether the anti-proliferative effect of REG on leukemia cells was associated with the induction of apoptosis, flow cytometric analysis was performed. As shown in Fig. 3, following exposure to REG at concentrations of 20,40 and $80 \mu \mathrm{g} / \mathrm{ml}$ for $24 \mathrm{~h}$, a concentration-dependent increase in apoptosis was observed. Thus, these results demonstrated that the cytotoxic activity of
REG on leukemia cells in vitro may be associated with the induction of apoptosis.

Effect of REG on the expression of caspase and Bcl-2 family proteins. In order to investigate the mechanism underlying the effect of REG, the levels of certain apoptosis-related proteins were detected. As shown in Fig. 4, the expression of cytochrome $c$, and c-caspases- 3 and -9 proteins was found to be significantly upregulated in a concentration-dependent manner in the REG treatment group compared with the control group $(\mathrm{P}<0.05)$. Furthermore, the expression levels of proteins from the Bcl-2 family were also determined in the present study (Fig. 5). The results showed that the Bcl-2 expression was significantly downregulated, whereas the expression of Bax was significantly upregulated compared with the control group $(\mathrm{P}<0.05)$.

In vivo anticancer effects of REG on the HL-60 xenograft model in nude mice. As shown in Fig. 6, following treatment with REG (40 mg/kg/day for 15 days), a significant inhibitory effect on tumor growth was observed during the 15-day observation period $(\mathrm{P}<0.05)$ compared with the control group. Furthermore, the expression of caspases-3 and -9 , Bax and $\mathrm{Bcl}-2$ proteins in the tumor tissues was examined. The results showed that the expression of c-caspases-3 and -9 , and Bax increased significantly, whereas the expression of Bcl-2 was found to be clearly decreased in the REG-treated mice compared with that in the control group $(\mathrm{P}<0.01)$.

\section{Discussion}

To the best of our knowledge, no studies have been conducted regarding the antitumor effect of REG on leukemia. To the 
A

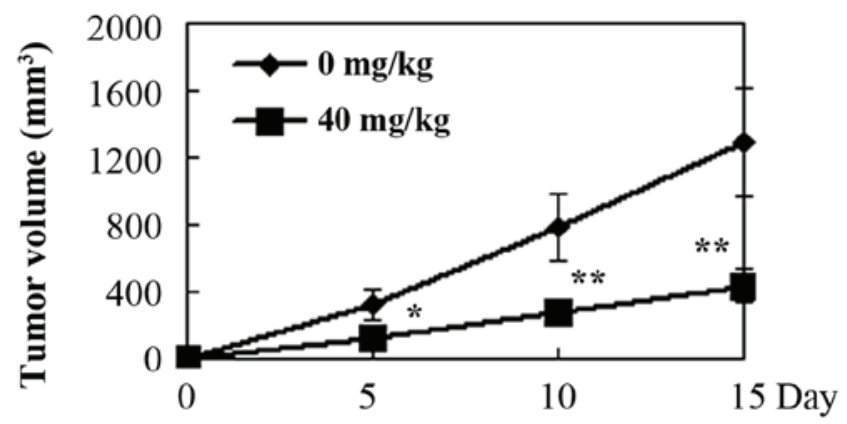

B
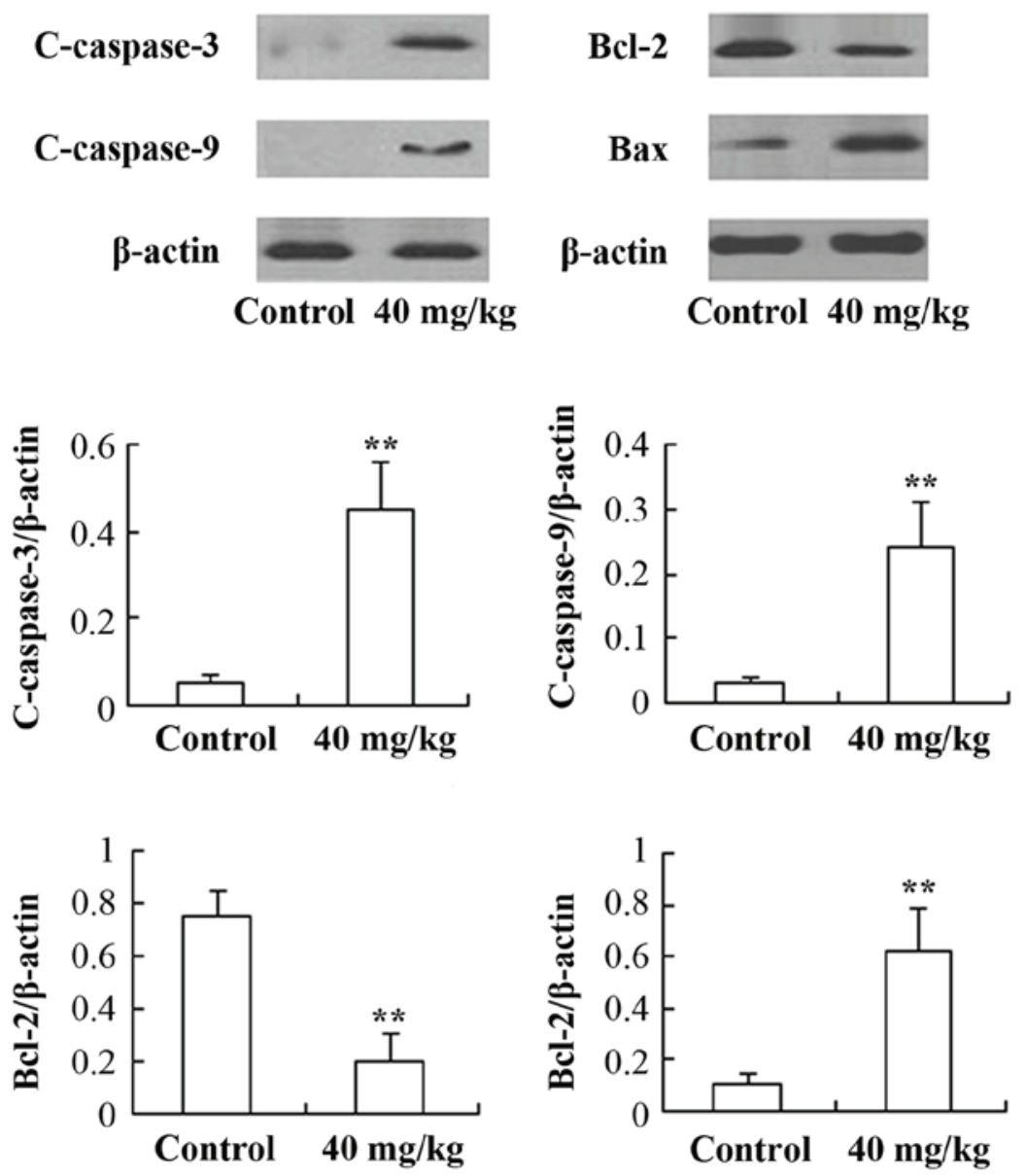

Figure 6. Antileukemia effects of REG in vivo. Effect of REG treatment on (A) tumor volume and (B) protein expression of c-caspases-3 and -9, Bax and Bcl-2 in tumor tissues. Data are presented as the mean \pm standard deviation $(\mathrm{n}=10)$. ${ }^{*} \mathrm{P}<0.05$ and ${ }^{* *} \mathrm{P}<0.01$ vs. control. REG, resveratrol-4-O-D-(2'-galloyl)-glucopyranoside; Bcl-2, B-cell lymphoma 2; Bax, Bcl-2-associated protein x; c-caspase, cleaved caspase.

best of our knowledge, the present study demonstrated for the first time that REG exerts a marked antileukemia activity, and that the underlying mechanism may be associated with the mitochondria-mediated apoptosis pathway.

It is known that apoptosis is a desirable pathway for killing cancer cells, and numerous studies have aimed to identify apoptosis-inducing agents (12-14). Mitochondria-mediated apoptosis has been reported to be an important apoptosis pathway (15). The release of cytochrome $c$ into the cytosol from the mitochondria is the initial step in the induction of apoptosis, and the apoptosis-associated caspase proteins, such as caspase-9. It has been reported that cytosolic cytochrome $c$ can activate procaspase- 9 by binding to the apoptotic peptidase activating factor- 1 in the presence of deoxyadenosine triphosphate, resulting in the activation of caspase-9 $(15,17)$. The activation of caspase-9 often leads to the activation of caspase-3. Caspase proteins are a family of cysteine proteases that are activated in the execution phase of apoptosis. Caspase- 3 is a crucial signaling protein of the mitochondria-mediated apoptosis pathway, and is considered to be the most important 'executioner' protein; therefore, the activation of caspase-3 is usually considered to be a biochemical hallmark of apoptosis $(18,19)$.

In the present study, it was demonstrated that REG treatment can significantly increase the cytochrome $c$ expression levels in the cytoplasm. In addition, the key caspase proteins, caspases-3 and -9, were also upregulated following exposure to REG. These results indicated that the anticancer activity of 
REG in leukemia cells could be associated with the mitochondria-mediated apoptosis pathway.

The Bcl-2 family of proteins is also considered to exhibit a key role in the regulation of the mitochondria-mediated apoptosis pathway. Bax is one of the main pro-apoptotic proteins of the Bcl-2 family, whereas Bcl-2 is one of the main anti-apoptotic proteins. Furthermore, the ratio of $\mathrm{Bax} / \mathrm{Bcl}-2$ is a key factor in the induction of mitochondrial-mediated apoptosis $(3,20)$. The results of the present study showed that Bcl-2 protein expression was markedly downregulated following REG treatment. By contrast, the expression level of Bax was found to be significantly increased following treatment with REG; therefore, the relative ratio of $\mathrm{Bax} / \mathrm{Bcl}-2$ was significantly elevated by $\mathrm{REG}$ treatment. In addition, the results of the in vivo experiment were consistent with the results obtained in vitro, suggesting that the anticancer activity of REG in leukemia is associated with the mitochondria-mediated apoptosis pathway.

In conclusion, the present study demonstrated that REG is an effective and reliable antileukemia agent, and that the underlying mechanism may be associated with the induction of the caspase-3 cascade, as well as the downregulation of the anti-apoptotic Bcl-2 family proteins. Findings from the current study indicated that REG exerts marked antitumor effects on leukemia cell lines, which may suggest the compound has potential clinical use as a therapeutic agent; however, further investigation of the mechanism underlying the effects of REG is required.

\section{Acknowledgements}

The present study was supported by grants from the National Science \& Technology Pillar Program during the 12th Five-year Plan Period (grant no. 2012BAI37B01) and the subtopic of Shanghai Committee of Science and Technology (grant no. 12DZ1941803).

\section{References}

1. Siegel R, Ma J, Zou ZH and Jemal A: Cancer Statistics, 2014. CA Cancer J Clin 64: 9-29, 2014.

2. Burnett AK, Goldstone A, Hills RK, Milligan D, Prentice A, Yin J, Wheatley K, Hunter A and Russell N: Curability of patients with acute myeloid leukemia who did not undergo transplantation in first remission. J Clin Oncol 31: 1293-1301, 2013.

3. Hseu YC, Lee CC, Chen YC, Kumar KJ, Chen CS, Huang YC, Hsu LS, Huang HC and Yang HL: The anti-tumor activity of Antrodia salmonea in human promyelocytic leukemia (HL-60) cells is mediated via the induction of $\mathrm{G}_{1}$ cell-cycle arrest and apoptosis in vitro or in vivo. J Ethnopharmacol 153: 499-510, 2014.
4. Chen HJ, Lo YC and Chiang WC: Inhibitory effects of adlay bran (Coix lachryma-jobi L. var. ma-yuen Stapf) on chemical mediator release and cytokine production in rat basophilic leukemia cells. J Ethnopharmacol 141: 119-127, 2012.

5. Lansky EP, Paavilainen HM, Pawlus AD and Newman RA: Ficus spp. (fig): Ethnobotany and potential as anticancer and anti-inflammatory agents. J Ethnopharmacol 119: 195-213, 2008.

6. Li JW and Vederas JC: Drug discovery and natural products: End of an era or an endless frontier? Science 325: 161-165, 2009.

7. Ovadje P, Chatterjee S, Griffin C, Tran C, Hamm C and Pandey S: Selective induction of apoptosis through activation of caspase- 8 in human leukemia cells (Jurkat) by dandelion root extract. J Ethnopharmacol 133: 86-91, 2011.

8. Peng W, Qin RX,Li XL andZhou H: Botany, phytochemistry, pharmacology, and potential application of Polygonum cuspidatum Sieb.et Zucc.: A review. J Ethnopharmacol 148: 729-745, 2013.

9. National Research Council (US) Committee for the Update of the Guide for the Care and Use of Laboratory Animals: Guide for the Care and Use of Laboratory Animals. 8th ed. National Academy Press, Washington, D.C., 2010.

10. Ngamwongsatit P, Banada PP, Panbangred W and Bhunia AK: WST-1-based cell cytotoxicity assay as a substitute for MTT-based assay for rapid detection of toxigenic Bacillus species using $\mathrm{CHO}$ cell line. J Microbiol Methods 73: 211-215, 2008.

11. Su YT, Chang HL, Shyue SK and Hsu SL: Emodin induces apoptosis in human lung adenocarcinoma cells through a reactive oxygen species-dependent mitochondrial signaling pathway. Biochem Pharmacol 70: 229-241, 2005.

12. Fuchs Y and Steller H: Programmed cell death in animal development and disease. Cell 147: 742-758, 2011.

13. Gao N, Budhraja A, Cheng SP, Yao H, Zhang Z and Shi XL: Induction of apoptosis in human leukemia cells by grape seed extract occurs via activation of c-Jun NH2-terminal kinase. Clin Cancer Res 15: 140-149, 2009.

14. Wu JG,Pen W, Yi J, Wu YB, Chen TQ and Wu JZ: Chemical composition, antimicrobial activity against Staphylococcus aureus and a pro-apoptotic effect in SGC-7901 of the essential oil from Toona sinensis (A. Juss.) Roem. leaves. J Ethnopharmacol 154: 198-205, 2014.

15. Hellwig CT, Passante E and Rehm M: The molecular machinery regulating apoptosis signal transduction and its implication in human physiology and pathophysiologies. Curr Mol Med 11: $31-47,2011$

16. Jiang $X$ and Wang $X$ : Cytochrome C-mediated apoptosis. Annu Rev Biochem 73: 87-106, 2004.

17. Zou H, Yang R, Hao J, Wang J, Sun C, Fesik SW, Wu JC, Tomaselli KJ and Armstrong RC: Regulation of the Apaf-1/caspase-9 apoptosome by caspase-3 and XIAP. J Biol Chem 278: 8091-8098, 2003.

18. Twiddy D and Cain K: Caspase-9 cleavage, do you need it? Biochemical J 405: e1-e2, 2007.

19. Woo HJ, Jun do Y, Lee JY, Woo MH, Yang CH and Kim YH: Apoptogenic activity of $2 \alpha, 3 \alpha$-dihydroxyurs-12-ene-28-oic acid from Prunella vulgaris var. lilacina is mediated via mitochondria-dependent activation of caspase cascade regulated by Bcl-2 in human acute leukemia Jurkat $\mathrm{T}$ cells. J Ethnopharmacol 135: 626-635, 2011.

20. Yang XK, Xu MY, Xu GS, Zhang YL and Xu ZX: In vitro and in vivo antitumor activity of scutebarbatine $A$ on human lung carcinoma A549 cell lines. Molecules 19: 8740-8751, 2014. 